\title{
EFFICIENT ZERO-KNOWLEDGE IDENTIFICATION SCHEME FOR SMART CARDS
}

\author{
Thomas Beth \\ Universität Karlsruhe \\ Fakultăt für Informatik \\ Institut für Algorithmen und Kognitive Systeme \\ Haid-und-Neu-Str. 7 \\ Technologie-Fabrik \\ D.7500 Karlsruhe
}

\section{ABSTRACT:}

In this paper we present a Fiat-Shamir like authentication protocol for the El-Gamal Scheme.

\section{Introduction}

The invention of the El-Gamal Scheme [1] has provided another Public-Key-Cryptosystem besides the renowned RSA-System, for which in addition to the Key-Exchange feature both Public-Key-Encryption and Signature Schemes are available. The availability of fast exponentiation hardware for the fields $G F\left(2^{n}\right)$, cf [2], [3] makes this algorithm very attractive for implementation in high-speed-communications. The recent invention of the Fiat-Shamir Authentication Protocol [4] has again attracted wide attention to the RSA-Scheme.

The purpose of this note is to show that a similar type of authentication protocol is available for the El-Gamal-Scheme based on the DiffieHellman One-Way-Function, with complexity, and/or error-probability considerably reduced as compared to the Fiat-Shamir-Scheme. 


\section{The Basic Protocol}

Suppose Alice (A) wants to authenticate herself to Bob (B). For this purpose $A$ has visited a trusted authority, which for obvious reasons we shall call the Secure Key lssuing Authority (SKIA).

\section{Initiation Phase}

The SKIA possesses secret logarithms $x_{1}, \ldots, x_{m}$, whose exponentiated values $y_{j}=\alpha^{x_{j}}$ are public. Here $\alpha$ is a primitive element of GF(q) known publicly. The SKIA also publishes the one-way-hashing function $f$.

\section{Setting-up Phase}

A goes to the SKIA, identifying herself by <names.

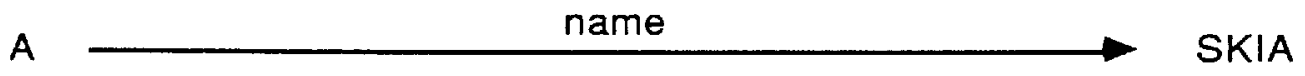

Then the SKIA produces $m$ identification numbers $I_{1}, \ldots, I_{m}$ for $A$ by using the public (random) one-way-function $\mathrm{f}$.

$$
\mathrm{ID}_{\mathrm{j}} \leftarrow \mathrm{f} \text { (name, j) }
$$

The SKIA chooses a (secret) random logarithm $k=k_{A}$ and forms

The SKIA also determines $m$ signatures $s_{j}$ as solutions of

$$
r \leftarrow \alpha^{k}
$$

$$
\text { (ID) } x_{j} r+k s_{j} \equiv I D_{j} \bmod (q-1) \text { for } j \in[1: m] \text {. }
$$

Eventually the SKIA issues a card (with secure memory) ${ }^{*}$ to A.

A

$$
s_{1}, \ldots, s_{m}
$$

) see sect. 5 


\section{Authentication Phase (Protocol Auth)}

A now approaches $B$ identifying herself by her name and the parameter $r$.

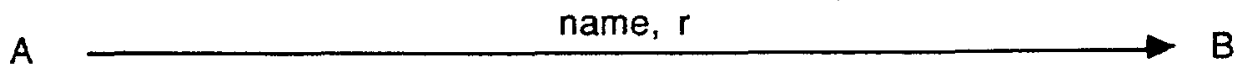

$B$ computes $A^{\prime}$ s identification numbers and the values $\rho_{1}, \ldots, \rho_{m}$

The following procedure is iterated for $i=1$ to $\mathrm{h}$ :

$$
\text { For } \begin{aligned}
& j \in[1: m]: 1 D_{j} \leftarrow f(\text { name, } j) \\
& \text { and } \rho_{j} \leftarrow y_{j}^{r}
\end{aligned}
$$

DO

A chooses a random element $t_{i} \in \mathbb{Z}_{q-1}$, forms

$z_{i} \leftarrow r^{-t_{i}}$

and sends it to $B$

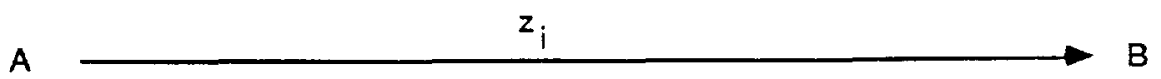

$B$ chooses a random string $b_{i}=\left(b_{i j}\right) \in R^{m}$ and sends it to $A$, where $R \subset \mathbb{Z}_{Q-1}$ is a suitably chosen subset

$\mathrm{b}_{\mathrm{i}}$

A computes

$$
u_{i} \leftarrow t_{i}+\sum_{j} b_{i j} s_{j} \bmod (q-1)
$$

and sends it to $B$
A
$u_{i}$
B computes

$$
\begin{aligned}
& v_{i}+\sum_{j} b_{i j} \cdot 1 D_{j} \\
& \gamma_{i} \leftarrow\left(\prod_{i} p_{j}^{b_{i j}}\right) \cdot r^{u_{i}} \cdot z_{i}-\alpha^{v_{i}}
\end{aligned}
$$


$B$ accepts the authenticity of $A$ if for all $i \in[1: h] \quad \gamma_{i}=0$. In this case we say that Protocol Auth ends successfully.

\section{Analysis of the Protocol}

\subsection{Observation (Verification)}

If $A$ and $B$ are genuine, the Protocol Auth ends successfully.

Proof: For all $i \in[1: \mathrm{h}]$

$$
\left(\prod_{j} \rho_{j} b_{i j}\right)
$$

can be computed by B based on B's knowledge.

From the definition we have

$$
\left(\prod_{j} \rho_{j}{ }^{b_{i j}}\right)=\prod_{j} y_{j}^{r b_{i j}}=\alpha^{r \sum_{j} x_{i j} b_{i j}}
$$

$B$ can also compute $r u_{i}$ from $u_{i} \cdot$ Having received $z_{i}$, by definition

$$
r^{u_{i}} \cdot z_{i}=r^{t_{i}+\sum_{i} b_{i j} s_{j}} \cdot r^{-t_{i}} . \text { Thus } \alpha^{\sum_{i} b_{i j}\left(r x_{i}+k s_{j}\right)}=\alpha^{v_{i}} \text {. }
$$

\subsection{Observation (Correctness)}

Assume, that $A$ cannot compute El-Gamal signatures in polynomial time: If $A$ is false, i.e. does not possess the signatures $s_{j}$, then the protocol Auth ends successfully with a false-right probability

$$
p_{1} \leq \frac{1}{|R|^{m \cdot h}}
$$

Proof: As long as $|R|$ is small enough as compared to $q^{m}$ (see remarks), A would have to guess the challenge vectors $\underline{b}_{j}$ in advance, analogously to the method described in the proof of lemma 2 by Fiat and Shamir [4]. 


\subsection{Remark}

The cheating method discussed in the proof of lemma 3.2 is only interesting if the size of choice space $R^{m}$ for the $\underline{b}_{i}$ is small compared to the complexity of forging El-Gamal signatures, which itself is at most as hard as taking discrete logs, cf. sect. 4.

\subsection{Lemma (Security)}

For arbitrary $q$ and $h$, with fixed $m$ and $|R| \in O\left((\log q)^{w}\right)$ for given $w \in \mathbb{N}$ the Protocol Auth is a Zero-Knowledge Protocol.

Proof: Following the papers by Berger/Kannan/Peralta [9] and Chaum, Evertse, van de Graaf [5] it can be seen that the size of the choice space $R^{m}$ is the decisive parameter for the construction of a poly-time-simulator $S$ for a cheating $B$ : to guarantee a probability for $S$ to "guess" the challenge $\underline{b}_{i}$ correctly in poly-time, we have to provide $|R|^{m}$ such that

$$
\text { prob ("badluck") }=\left(1-\frac{1}{|R|^{m}}\right)^{\text {poly }(\log (q))}<c^{\log q}
$$

for some constant $c>1$.

\section{Practical Security Considerations}

The system (ID) gives $m$ linear equations for $(m+1)$ unknowns (w.r.t. the assumption that the discrete log problem is unfeasible). As consequences we note:

(i) Not even A can forge new signatures.

(ii) The requirement of storing the signatures $s_{j}$ in the secure memory of the card is only needed as protection against copying the card.

(iii) This requirement could be dropped if the one-way-hash function $f$ (when stored on the card) could be employed by the card as a means of testing the user's identity before the card is authenticated. For this test several user features can be challenged, in each case requiring an interface between user and card, however!

(iv) To bring the security of the signatures closer to the level of the discrete log-problem it may be feasible to make the computation of the $I D_{j}$ additionally dependent on the public random number $r$. 


\section{Implementation Aspects}

In view of the demand for low cost designs of security processors for chip cards we suggest considering the following case for practical implementation :

$$
q=2^{n} \text {, }
$$

where $n$ should be suitably chosen, roughly in the interval $\left[2^{9}: 2^{11}\right]$ depending on the required security. For these cases fast VLSI exponentiators have been suggested (Beth/Cook/Gollmann [3], Vanstone/ Mullin [2], Massey/Omura/Wang [7]).

For $\mathrm{q}=2^{\mathrm{n}}$ the Discrete Log Problem can be solved in

$$
O\left(\exp \left(c \cdot n^{1 / 3} \cdot(\log n)^{2 / 3}\right)\right.
$$

steps [6]. Therefore a suitable amount of security can be guaranteed.

\subsection{Tuning the Protocol}

Using the fact that squaring is a field automorphism in $G F\left(2^{n}\right)$ we suggest to use the following refinements of the protocol in order to save on computational effort and required storage area as well as on the length $h$ of the protocol:

*) Choose the random string only from binary words of weight less than $w$, i.e. choose $b_{i j}$ equally distributed in

$$
\left.R=\left\{b \in Z_{q-1} \mid \text { wgt (binary }(b)\right) \leq w\right\}
$$

\subsubsection{Corollary}

With these additional restrictions if $A$ is false the protocol ends successfully with probability

$$
p_{1} \leq \frac{1}{2^{1 \cdot m \cdot h}}
$$

where

$$
t=\log \left(\sum_{i=0}^{w}\left(\begin{array}{l}
n \\
i
\end{array}\right)\right)
$$


**) Choosing the further simplification $m=1$, the number of computational steps especially in computing

$$
\gamma_{i} \leftarrow p^{\sum_{i=1}^{m} b_{i j}} \cdot r^{u_{i}} \cdot z_{i}-\alpha^{v_{i}}
$$

is reduced considerably.

***) Combining $(* *)$ and $(*)$ for $w=1$ the exponent of $\rho$ being a power of two requires a fast squaring operation only.

\subsubsection{Technical Observation}

With $m=1$ and $h=3$ and $\log q>2^{9}$ the Protocol Auth allows an authentication procedure at a residual false-right error probability smaller than

$$
\begin{array}{lll}
10^{-8} & \text { for } & w=1 \\
10^{-15} & \text { for } & w=2 \\
10^{-22} & \text { for } & w=3
\end{array}
$$

\subsection{Conclusion}

With one signature $(m=1)$ and a small number of iterations $(1 \geq 3)$ this protocol provides a security level appropriate to many smart card applications. In comparison to Fiat-Shamir's protocol [4] the memory consumption on the smart card is considerably reduced for the proposed protocol, as the signature $s_{j}$ and the number $r$ only require approximately 64 Bytes each, and the representations of $G F\left(2^{n}\right)$-arithmetics can be compressed to considerably less bits. If the application requires only to authenticate the card through a trusted terminal, the public keys $y$ need not to be stored on the card. Otherwise, the same protocol of course would be used by $A$ to challenge $B$.

Note that an additional advantage to this protocol is provided by the fact, that based on purpose-made-algorithms the $G F\left(2^{n}\right)$-arithmetics can be carried out at a higher speed than modular arithmetic required for the Fiat-Shamir-Scheme. 
In summary, the present scheme provides a user-friendly zero-knowledge authentication and signature protocol that offers itself as a small, fast and low cost verification tool for the use in token technology as it is presented by smart cards, intelligent tokens and other identification mechanisms.

\section{Acknowledgement}

The author is grateful to Dr. Ivan Damgárd for his helpful critical remarks.

\section{References}

[1] El-Gamal: A Public Key Cryptosystem and a Signature Scheme Based on Discrete Logarithms, IEEE-IT-31, 469-472, 1985

[2] Vanstone, Mullin: Communication 1986, Cryptech, Waterbo, Ontario, Canada

[3] Beth, Cook, Gollmann: Architectures for Exponentiation in GF(2n), Proceedings of Crypto 86, Santa Barbara, Springer LNCS 263, 302-310, 1987

[4] Fiat, Shamir: How to prove yourself: Practical solutions to identification and signature problems, Proceedings of Crypto 86, Santa Barbara, Springer LNCS 263, 186-194, 1987

[5] Chaum, Evertse, van de Graaf: An unproved Protocol for Demonstrating Possession of Discrete Logarithms and some Generalizations, Proceedings EUROCRYPT'87, Springer LNCS 304, 127-141, 1988

[6] Coppersmith: Fast Evaluation of Logarithms in Fields of Characteristic Two, IEEE-IT-30, 587-594, 1984

[7] Wang: Exponentiation in Finite Fields, Ph.D. dissertation , University of California, Los Angeles, 1985

[8] Goldwasser, S.: Micali, S.; Rackoff, C.: The Knowlege Complexity of Interactive Proof Systems, Proc. 17th ACM Symp. on Theory of Computing, 1985

[9] Berger, Kannan, Peralta: A Framework for the Study of Cryptographic Protocols, Proc. CRYPTO'85, Springer LNCS 218, 87-103 